\title{
THE HURST INDEX OF LONG-RANGE DEPENDENT RENEWAL PROCESSES
}

\author{
By D. J. DALEY \\ Australian National University
}

\begin{abstract}
A stationary renewal process $N(\cdot)$ for which the lifetime distribution has its $k$ th moment finite or infinite according as $k$ is less than or greater than $\kappa$ for some $1<\kappa<2$, is long-range dependent and has Hurst index $\alpha=\frac{1}{2}(3-\kappa)$ (this is the critical index $\alpha$ for which $\lim \sup _{t \rightarrow \infty} t^{-2 a} \operatorname{var} N(0, t]$ is finite or infinite according as $a$ is greater than or less than $\alpha$ ). This identification is accomplished by delineating the growth rate properties of the difference between the renewal function and its linear asymptote, thereby extending work of Täcklind.
\end{abstract}

1. Introduction and statement of results. The question stimulating this note concerns the identification of the Hurst index of a long-range dependent (LRD) stationary renewal process (see below for definitions). Answering this question led us to revisit work of Täcklind on the order of the difference between the renewal function and its linear asymptote when a generic lifetime of the renewal process has infinite second moment, which is a necessary and sufficient condition for the stationary renewal process to be LRD.

Historically [we refer the reader to Beran (1994) for a broad account] longrange dependent processes $\left\{X_{n}\right\}$ in discrete time evolved in work of the hydrologist Hurst (1951) who studied annual flow data of the River Nile. This motivated Mandelbrot and coworkers to introduce fractional Gaussian noise as a statistical model with long memory. Landmarks in the statistical literature include Taqqu (1986) and, especially, Beran (1994). Adaptation of the idea to the point process setting, for second-order stationary point processes, is in Daley and Vesilo (1997).

A stationary renewal process with counting function $N(A)$ denoting the number of points in the Borel set $A$ is characterized by the distribution function (d.f.) $F$ of a generic lifetime random variable (r.v.) $X$ which has finite mean $\lambda^{-1}=\mathrm{E}(X)=\int_{0}^{\infty} x d F(x)$. For convenience we assume that $0=F(0+)=$ $\operatorname{Pr}\{X=0\}$ and write $N(0, t]$ when $A$ is the half-open interval $(0, t] . N$ is longrange dependent $(\mathrm{LRD})$ in the sense that

$$
\limsup _{t \rightarrow \infty} \frac{\operatorname{var} N(0, t]}{t}=\infty
$$

if and only if $\mathrm{E}\left(X^{2}\right)=\infty$, because this is the necessary and sufficient condition for the integrand in the representation [e.g., Daley and Vere-Jones (1988),

Received November 1998; revised July 1999.

AMS 1991 subject classifications. Primary 60K05; secondary 60G55.

Key words and phrases. Hurst index, long-range dependence, renewal process, renewal function asymptotics, regular variation, moment index. 
Chapter 3, Section 5 and equation (4.3.4)]

$$
V(t) \equiv \operatorname{var} N(0, t]=\lambda \int_{0}^{t}(2[U(u)-\lambda u]-1) d u
$$

to diverge to $\infty$ as $t \rightarrow \infty$ [e.g., Täcklind (1944), Feller (1966), Chapter XI, Section 4, or (1971), Chapter XI, Section 3]; in (1.2), $U(t) \equiv \sum_{n=0}^{\infty} F^{n *}(t)$, where $F^{n *}$ is the $n$-fold convolution of $F$ with itself, is the renewal function; recall the elementary renewal theorem $U(t) / t \rightarrow \lambda(t \rightarrow \infty)$.

A stationary point process $N(\cdot)$ with finite second moment necessarily has $\mathrm{E}\left((N(0, t])^{2}\right)<C t^{2}$ (all $\left.t \geq 1\right)$ for some finite $C$, so its Hurst index $\alpha$ is well defined by

$$
\alpha \equiv \inf \left\{a: \limsup _{t \rightarrow \infty} \frac{\operatorname{var} N(0, t]}{t^{2 a}}<\infty\right\}
$$

and lies in the interval $0 \leq \alpha \leq 1$. For a LRD renewal process, it follows from (1.2) and the subadditivity of the renewal function $U(t+u) \leq U(t)+U(u)$ $(t, u>0)$ [e.g., Daley (1978)] that $U(t) \geq \lambda t$ and hence that $V(t) \geq \lambda t$ also, and so its Hurst index lies in the interval $\left[\frac{1}{2}, 1\right]$. Indeed, in terms of its order for large $t$, the variance function $V$ has the same asymptotic behavior as the function

$$
\widetilde{V}(t) \equiv V(t)+\lambda t=2 \lambda \int_{0}^{t}[U(u)-\lambda u] d u .
$$

The characterization we give of the Hurst index of a stationary LRD renewal process is in terms of the moment index

$$
\kappa \equiv \sup \left\{k: \mu_{k} \equiv \mathrm{E}\left(X^{k}\right)=\int_{0}^{\infty} x^{k} d F(x)=\int_{0}^{\infty} x^{k-1} \bar{F}(x) d x<\infty\right\}
$$

where $\bar{F}(v)=1-F(v)(v \geq 0)$ [in (1.5), $k$ is any real greater than or equal to 0].

THEOREM 1. A stationary renewal process with lifetime distribution function $F$ which has $\int_{0}^{\infty} x^{2} d F(x)=\infty, \int_{0}^{\infty} x d F(x)<\infty$ and moment index $\kappa$, is long-range dependent and has Hurst index $\alpha=\frac{1}{2}(3-\kappa)$.

The new part of this statement is the identification of the Hurst index. This identification has certainly been known in cases such as those where the lifetime d.f. $F$ has a regularly varying tail, when rather more is then known as in Example 1.

EXAMPLE 1 (Renewal process whose lifetime d.f. has a regularly varying tail.) From, for example, Feller [(1971), Chapter XI, Section 3 or Bingham, Goldie and Teugels (1987), Section 8.6.1], the lifetime d.f. $F$ has a regularly varying tail, meaning

$$
1-F(x) \sim x^{-\kappa} l(x), \quad x \rightarrow \infty,
$$


where $1<\kappa<2$ and $l(\cdot)$ is any slowly varying function, if and only if the discrepancy function $\phi(t) \equiv U(t)-\lambda t$ satisfies

$$
\phi(t) \sim \lambda^{2} t^{2-\kappa} l(t) /[(2-\kappa)(\kappa-1)], \quad t \rightarrow \infty .
$$

Since regular variation is preserved under integration [Bingham, Goldie and Teugels (1987), Proposition 1.5.8], it follows from (1.4) that when (1.6) holds,

$$
\tilde{V}(t)=2 \lambda \int_{0}^{t} \phi(u) d u \sim \frac{2 \lambda^{3} t^{3-\kappa} l(t)}{(3-\kappa)(2-\kappa)(\kappa-1)}, \quad t \rightarrow \infty
$$

From these relations we can read off a range of possible behaviors for $\tilde{V}(t)$, with $\alpha=\frac{1}{2}(3-\kappa)$ in all cases, consistent with Theorem 1 :

1. If $\mu_{\kappa}<\infty$ then $\int_{0}^{\infty} x^{-1} l(x) d x<\infty$ and hence $l(x) \rightarrow 0(x \rightarrow \infty)$ because $l$ is slowly varying [Proposition 1.5.9b of Bingham, Goldie and Teugels (1987)]. Then $\tilde{V}(t)=o\left(t^{3-\kappa}\right)(t \rightarrow \infty)$ in this case.

2. We can have both $\mu_{\kappa}=\infty$ and $l(x) \rightarrow 0$, so that $\tilde{V}(t)=o\left(t^{3-\kappa}\right)$ is possible when $\mu_{\kappa}=\infty$.

3. We can also have both $\mu_{\kappa}=\infty$ and $l(x) \rightarrow \infty$ for large $x$, in which case $\widetilde{V}(t)>O\left(t^{3-\kappa}\right)$, though $\tilde{V}(t)=o\left(t^{3-\kappa+\varepsilon}\right)$ for arbitrarily small positive $\varepsilon$.

Our method of proof enables us to recover Täcklind's result that $\phi(t)=$ $U(t)-\lambda t=o\left(t^{2-\kappa}\right)$ when $\mu_{\kappa}<\infty$ and $\kappa<2$ and to elucidate further properties of the function $\phi(t)$ as in Theorem 2. Like Täcklind, our methods are wholly real variable, and do not involve any transform calculus. Example 2 in Section 3 shows that we cannot weaken the result.

THEOREM 2. For a renewal process with finite mean lifetime $\lambda^{-1}$, infinite second moment and moment index $\kappa$, as $t \rightarrow \infty$ and for arbitrarily small positive $\varepsilon$,

$$
U(t)-\lambda t \begin{cases}=o\left(t^{2-\kappa}\right), & \mu_{\kappa}<\infty \text { and } \kappa<2 \\ <o\left(t^{2-\kappa+\varepsilon}\right), & \mu_{\kappa}=\infty\end{cases}
$$

and in either case,

$$
\limsup _{t \rightarrow \infty} \frac{U(t)-\lambda t}{t^{2-\kappa-\varepsilon}}=\infty
$$

Note that in the latter case of (1.9), it follows from a result of Vuilleumier at Theorem 2.3.6 of Bingham,Goldie and Teugels (1987) that there exists a slowly varying function $l(\cdot)$ such that

$$
[U(t)-\lambda t] /\left[t^{2-\kappa} l(t)\right] \rightarrow 0, \quad t \rightarrow \infty
$$


2. Proofs. It is a standard result [e.g., Feller (1966), Chapter XI, Section 4] that the discrepancy function $\phi(t) \equiv U(t)-\lambda t$ satisfies the equation

$$
\phi(t)=\lambda \int_{t}^{\infty} \bar{F}(v) d v+\int_{0}^{t} \phi(t-u) d F(u),
$$

and hence, from the solution of such a general renewal equation [e.g., Feller (1966) Chapter XI, equation (1.4)],

$$
\begin{aligned}
\phi(t) & =\int_{0}^{t} d U(u) \int_{t-u}^{\infty} \bar{F}(v) d v \\
& =\int_{0}^{t}[U(t)-U(t-v)] \bar{F}(v) d v+U(t) \int_{t}^{\infty} \bar{F}(v) d v . \\
& \leq \int_{0}^{\infty} U(\min (v, t)) \bar{F}(v) d v \quad \text { by subadditivity of } U .
\end{aligned}
$$

Now the elementary renewal theorem implies that $U(t)<A+B t$ (all $t \geq 0$ ) for some positive $A$ and $B$, so

$$
\begin{aligned}
\frac{\phi(t)}{t^{2-\kappa}} \leq & \frac{A}{t^{2-\kappa}} \int_{0}^{\infty} \bar{F}(v) d v \\
& +\int_{0}^{\infty} B \min \left[\left(\frac{t}{v}\right)^{\kappa-1},\left(\frac{v}{t}\right)^{2-\kappa}\right] v^{\kappa-1} \bar{F}(v) d v .
\end{aligned}
$$

When $\infty>\mu_{\kappa}=\int_{0}^{\infty} v^{\kappa-1} \bar{F}(v) d v$, we can appeal to the dominated convergence theorem to conclude that the second integral in (2.3) $\rightarrow 0$ as $t \rightarrow \infty$, while the term involving the other integral approaches 0 by inspection. This proves Täcklind's result, the first part of (1.9).

Irrespective of the finiteness of $\mu_{\kappa}$, we have $\mu_{\kappa-\varepsilon}<\infty$ for positive $\varepsilon<\kappa$ and so for arbitrarily small positive $\varepsilon$, we have by a similar argument that for $0<\varepsilon<\kappa-1$, as $t \rightarrow \infty$,

$$
\begin{aligned}
\frac{\phi(t)}{t^{2-\kappa+\varepsilon}} \leq & \frac{A}{t^{2-\kappa+\varepsilon}} \int_{0}^{\infty} \bar{F}(v) d v \\
& +\int_{0}^{\infty} B \min \left[\left(\frac{t}{v}\right)^{\kappa-1-\varepsilon},\left(\frac{v}{t}\right)^{2-\kappa+\varepsilon}\right] v^{\kappa-1-\varepsilon} \bar{F}(v) d v \rightarrow 0 .
\end{aligned}
$$

Both parts of (1.9) are proved, and by substitution in (1.4) it follows that $\alpha \leq \frac{1}{2}(3-\kappa)$. To prove (1.10) and that $\alpha \nless \frac{1}{2}(3-\kappa)$, we argue by contradiction.

Suppose that (1.10) does not hold, that is, that for some positive $\varepsilon$ and $C$ as above (1.3), $\phi(t) \leq C t^{2-\kappa-\varepsilon}$ for all sufficiently large $t$. Then for finite positive $T$,

$$
\int_{T}^{\infty} \frac{\phi(t)}{t^{3-\kappa-\varepsilon / 2}} d t \leq \int_{T}^{\infty} t^{-1-\varepsilon / 2} d t \rightarrow 0, \quad T \rightarrow \infty .
$$

From the equality in (2.2) and the inequality $U(t) \geq \lambda t$, this integral certainly dominates

$$
\int_{T}^{\infty} \frac{\lambda}{t^{2-\kappa-\varepsilon / 2}} d t \int_{t}^{\infty} \bar{F}(v) d v=\int_{T}^{\infty} \frac{\left[v^{\kappa-1+\varepsilon / 2}-T^{\kappa-1+\varepsilon / 2}\right] \bar{F}(v)}{\kappa-1+\varepsilon / 2} d v=\infty
$$


by definition of $\kappa$. Hence a contradiction, so (1.10) must hold, and Theorem 2 is proved.

Just as $\phi(\cdot)$ satisfies a general renewal equation, so, too, does $\tilde{V}(\cdot)$ defined at (1.4), namely,

$$
\begin{aligned}
\tilde{V}(t) & =2 \lambda^{2} \int_{0}^{t} d s \int_{s}^{\infty} \bar{F}(v) d v+2 \lambda \int_{0}^{t} d s \int_{0}^{s} \phi(s-u) d F(u) \\
& =2 \lambda^{2}\left[\int_{0}^{t} v \bar{F}(v) d v+t \int_{t}^{\infty} \bar{F}(v) d v\right]+\int_{0}^{t} \tilde{V}(t-u) d F(u)
\end{aligned}
$$

so, appealing again to the solution of the general renewal equation as for (2.1),

$$
\begin{aligned}
\tilde{V}(t) & =2 \lambda^{2} \int_{0}^{t} d U(u) \int_{0}^{t-u} d s \int_{s}^{\infty} \bar{F}(v) d v \\
& =2 \lambda^{2} \int_{0}^{t} d s \int_{0}^{t-s} d U(u) \int_{s}^{\infty} \bar{F}(v) d v=2 \lambda^{2} \int_{0}^{t} U(t-s) d s \int_{s}^{\infty} \bar{F}(v) d v \\
& =2 \lambda^{2} \int_{0}^{t} U(s) d s \int_{t-s}^{\infty} \bar{F}(v) d v=2 \lambda^{2} \int_{0}^{\infty} \bar{F}(v) d v \int_{(t-v)_{+}}^{t} U(s) d s \\
& \geq \lambda^{3}\left[\int_{0}^{t} v(2 t-v) \bar{F}(v) d v+t^{2} \int_{t}^{\infty} \bar{F}(v) d v\right] \quad \text { since } U(s) \geq \lambda s .
\end{aligned}
$$

Suppose now that $\alpha<\frac{1}{2}(3-\kappa)$, so that for some positive $\varepsilon, \tilde{V}(t)<C t^{3-\kappa-\varepsilon}$ for all sufficiently large $t$ and some finite positive $C$. Then, much as at (2.5), for sufficiently large positive $T$,

$$
\int_{T}^{\infty} \frac{\tilde{V}(t)}{t^{4-\kappa-\varepsilon / 2}} d t \leq C \int_{T}^{\infty} t^{-1-\varepsilon / 2} d t \rightarrow 0, \quad T \rightarrow \infty
$$

However, the left-hand side here certainly dominates

$$
\begin{gathered}
\int_{T}^{\infty} \frac{\lambda^{3}}{t^{2-\kappa-\varepsilon / 2}} \int_{t}^{\infty} \bar{F}(v) d v= \\
\lambda^{3} \int_{T}^{\infty} \frac{\left[v^{\kappa-1+\varepsilon / 2}-T^{\kappa-1+\varepsilon / 2}\right] \bar{F}(v)}{\kappa-1+\varepsilon / 2} d v=\infty,
\end{gathered}
$$

the contradiction again coming from the definition of $\kappa$. So we cannot have $\alpha<\frac{1}{2}(3-\kappa)$, and Theorem 1 is proved.

\section{Example and discussion.}

EXAMPLE 1. This example shows that within the class of d.f.'s with specified moment index $\kappa$, we cannot refine the conclusions of Theorems 1 and 2, such as occurs in the context of Example 1, unless we impose some further condition on the d.f. $F$. Specifically, we describe a distribution for which (cf. Theorem 2) $\liminf \operatorname{in}_{t \rightarrow \infty} \phi(t) / t^{2-\kappa}=0$, $\limsup _{t \rightarrow \infty} \phi(t) / t^{2-\kappa}=\infty$. A similar example can be constructed for Theorem 1 . The genesis of the particular example is in the Example to Case I of Theorem 2.6.6 in Bingham, Goldie and Teugels (1987). 
Consider a lifetime distribution $F$ that consists of atoms of size $C p_{n} / x_{n}^{3 / 2}$ located at the points $x_{n}, n=1,2, \ldots$, where these points and the weights $p_{n}$ are so chosen that $p_{n} \rightarrow \infty(n \rightarrow \infty)$ but $\sum_{n=1}^{\infty} p_{n} / x_{n}^{\varepsilon}<\infty$ for every positive $\varepsilon$, no matter how small, and $C$ is a normalizing constant. Then the distribution $\left\{C p_{n} / x_{n}^{3 / 2}\right\}$ has moment index $\kappa=\frac{3}{2}$. In the setting of Theorem 2 we recall (2.2), so we are interested in

$$
\begin{aligned}
\frac{\phi(t)}{t^{1 / 2}} & \sim \frac{1}{t^{1 / 2}} \int_{0}^{v=t} \bar{F}(v) d\left(\frac{1}{2} v^{2}\right)+t^{1 / 2} \int_{t}^{\infty} \bar{F}(v) d v \\
& =\frac{1}{2} \int_{0}^{t}\left(\frac{v}{t}\right)^{1 / 2} v^{3 / 2} d F(v)+\frac{1}{2} \int_{t}^{\infty}\left(\frac{t}{v}\right)^{1 / 2}\left(2-\frac{t}{v}\right) v^{3 / 2} d F(v) .
\end{aligned}
$$

For the discrete distribution we are considering, these two integrals yield finite and infinite sums, respectively. For given $t$, we can find $n$ such that $x_{n}<t \leq$ $x_{n+1}$. Suppose the $\left\{p_{n}\right\}$ are such that the two sums at 3.1 are dominated by the terms in $p_{n}$ and $p_{n+1}$, respectively; a sufficient condition for this is that $x_{n+1} /\left(x_{n} p_{n}\right) \rightarrow \infty$ sufficiently fast. Then for such $t$ we have

$$
\frac{2 \phi(t)}{C t^{1 / 2}} \sim\left(\frac{x_{n}}{t}\right)^{1 / 2} p_{n}+\left(\frac{t}{x_{n+1}}\right)^{1 / 2}\left(2-\frac{t}{x_{n+1}}\right) p_{n+1} .
$$

This expression approaches $\infty$ for $t$ close to either $x_{n}$ or $x_{n+1}$, and the expression approaches 0 for $t \approx \sqrt{x_{n} x_{n+1}}$. When $p_{n}=n$ and $x_{n}=\exp \left(n^{2}\right)$ the sequences $\left\{p_{n}\right\}$ and $\left\{x_{n}\right\}$ satisfy the conditions we require.

This counterexample and, indeed, Theorem 1 (which can be phrased in the form of Theorem 2), suggest why the "verification" of long-range dependence and estimation of $\alpha$ can be fraught with difficulties. Suppose $\lambda=1$ and $\kappa=1.5$, hence $\alpha=0.75$. Then, if instead of estimating (and plotting) $\log \left(\tilde{V}(t) / t^{1.5}\right)$, we use the index 1.52 , the function is multiplied by $t^{0.02}=\exp (0.02 \log t) \approx$ $1+0.02 \log t$ when the exponent is small, such as $t=e^{14} \sim 10^{6}$; that is, the error need not be detectable using a million observations (and even with a billion $=10^{9} \approx e^{21}$ the same conclusion may hold).

Acknowledgments. This work arose out of discussion with Rein Vesilo concerning a sequel to Daley and Vesilo (1997). I thank him and Nick Bingham for discussion.

\section{REFERENCES}

BerAN, J. (1994). Statistics for Long-Memory Processes. Chapman and Hall, New York.

Bingham, N. H., Goldie, C. M. and Teugels, J. L. (1987). Regular Variation. Cambridge Univ. Press.

DALEY, D. J. (1978). Upper bounds for the renewal function via Fourier theoretic methods. Ann. Probab. 6 876-884.

DALEy, D. J. and VERE-JONES, D. (1988). An Introduction to the Theory of Point Processes. Springer, New York.

DALEY, D. J. and Vesilo, R. (1997). Long range dependence of point processes, with queueing examples. Stochastic Process Appl. 70 265-282.

FELleR, W. (1966). An Introduction to Probability Theory and Its Applications 2. Wiley, New York. 
FELleR, W. (1971). An Introduction to Probability Theory and Its Applications 2, 2nd ed. Wiley, New York.

HuRst, H. E. (1951). Long-term storage capacity of reservoirs. Trans. Amer. Soc. Civil Engineers $116770-799$.

TÄCKLIND, S. (1944). Elementare Behandlung vom Erneuerungsproblem für den stationären Fall. Skand. Aktuarietidskr. 27 1-15.

TAQQU, M. S. (1986). A bibliographical guide to self-similar processes and long-range dependence. In Dependence in Probability and Statistics (E. Eberlein and M. S. Taqqu, eds.) 137165. Birkhauser, Boston.

School of Mathematical Sciences AUSTRALIAN NATIONAL UNIVERSITY CANBERRA A.C.T. 0200

Australia

E-mail: daryl@maths.anu.edu.au 